\title{
One Dimensional Relativistic Free Particle in a Quadratic Dissipative Medium
}

\author{
G. V. López, G. C. Montes, J. G. T. Zanudo \\ Departamento de Fsica, Universidad de Guadalajara, Guadalajara, México \\ Email: gulopez@cencar.udg.mx
}

Received 26 January 2015; accepted 13 February 2015; published 16 February 2015

Copyright (C) 2015 by authors and Scientific Research Publishing Inc.

This work is licensed under the Creative Commons Attribution International License (CC BY). http://creativecommons.org/licenses/by/4.0/

c) (i) Open Access

\begin{abstract}
The deduction of a constant of motion, a Lagrangian, and a Hamiltonian for relativistic particle moving in a dissipative medium characterized by a force which depends on the square of the velocity of the particle is done. It is shown that while the trajectories in the space $(x, v)$, defined by the constant of motion, look as one might expected, the trajectories in the space $(x, p)$, defined by the Hamiltonian, have an odd behavior.
\end{abstract}

\section{Keywords}

\section{Lagrangian, Hamiltonian, Constant of Motion, Dissipation, Relativistic}

\section{Introduction}

It is well known that the Lagrangian and Hamiltonian approaches for some non-dissipative and some dissipative systems have some problems [1]-[6]. One of these problems consists of the possibility of having two different Hamiltonian to the same classical system [7], implying that one will have two different quantizations for this system. Another problem consists that for some dissipative non-relativistic systems, like a free particle moving in a dissipative medium characterized by a force which depends on the square of the velocity of the particle, the trajectories on the space $(x, p)$ have an odd behavior. However, the trajectories on the space $(x, v)$, defined by the constant of motion, have a good expected behavior [8]. Nevertheless, the interest in having Hamiltonian for dissipative system continues [9] [10].

In this work, the study of this former problem is extended to the relativistic motion of the particle. The constant of motion, the Lagrangian, and the Hamiltonian are deduced consistently, and it is shown that the behaviors of the trajectories of the particle in the phase space $(x, p)$ are odd when the Hamiltonian approach is used. However, the trajectories in the space $(x, v)$, when the constant of motion is used, behave as one can 
expected .

\section{Constant of Motion, Lagrangian and Hamiltonian}

The one-dimensional motion of a relativistic particle of mass " $m$ " at rest which is moving with a velocity $\dot{x}=\mathrm{d} x / \mathrm{d} t$ in a dissipative medium characterized by a force which depends on the square of this velocity is described by the equation

$$
\frac{\mathrm{d}}{\mathrm{d} t}(m \gamma \dot{x})=-\alpha \dot{x}^{2}
$$

where $\alpha$ is the dissipative parameter, $c$ is the speed of light, and $\gamma$ is the relativistic factor, $\gamma=\left(1-\dot{x}^{2} / c^{2}\right)^{-1 / 2}$. Actually, Equation (1) represents a dissipative system for $\dot{x} \geq 0$, otherwise it represents an anti-dissipative system. Therefore, only the case $\dot{x} \geq 0$ will be considered below. This system can be written as the following dynamical system

$$
\dot{x}=v, \quad \dot{v}=-\frac{\alpha v^{2}}{m}\left(1-v^{2} / c^{2}\right)^{3 / 2} .
$$

A constant of motion for this system is a function $K=K(x, v)$ such that it satisfies the following partial differential equation of first order [11]

$$
v \frac{\partial K}{\partial x}-\frac{\alpha v^{2}}{m}\left(1-v^{2} / c^{2}\right)^{3 / 2} \frac{\partial K}{\partial v}=0 .
$$

The general solution of this equation [12] is given by $K=G(C)$, where $G$ is an arbitrary function of the characteristic curve $C$,

$$
C=\frac{\alpha}{m} x+\frac{1}{\sqrt{1-v^{2} / c^{2}}}-\ln \left(\frac{1+\sqrt{1-v^{2} / c^{2}}}{v / c}\right) .
$$

By choosing $K=m c^{2} C$, a constant of motion is gotten with energy units,

$$
K=\alpha c^{2} x+\frac{m c^{2}}{\sqrt{1-v^{2} / c^{2}}}-m c^{2} \ln \left(\frac{1+\sqrt{1-v^{2} / c^{2}}}{v / c}\right) .
$$

The Lagrangian of the system can be consistently deduced from the known expression

$$
L(x, v)=v \int \frac{K(x, v)}{v^{2}} \mathrm{~d} v,
$$

which establishes the relation between the Lagrangian and the constant of motion of the system [13]-[16]. Using this expression it follows that

$$
L=-\alpha c^{2} x-2 m c^{2} \sqrt{1-v^{2} / c^{2}}+m c^{2} \ln \left(\frac{1+\sqrt{1-v^{2} / c^{2}}}{v / c}\right) .
$$

The generalized linear momentum, $\quad p=\partial L / \partial v$, is given by

$$
p=m c \frac{2 v^{2} / c^{2}-1}{(v / c) \sqrt{1-v^{2} / c^{2}}} .
$$

The plot of this expression and the plot of the usual relativistic free linear momentum expression $\left(p=m v / \sqrt{1-v^{2} / c^{2}}\right)$ are shown in Figure 1, where one sees that for Equation (8) there is not a one to one relation between the velocity $v$ and the generalized linear momentum $p$ of Equation (8). 


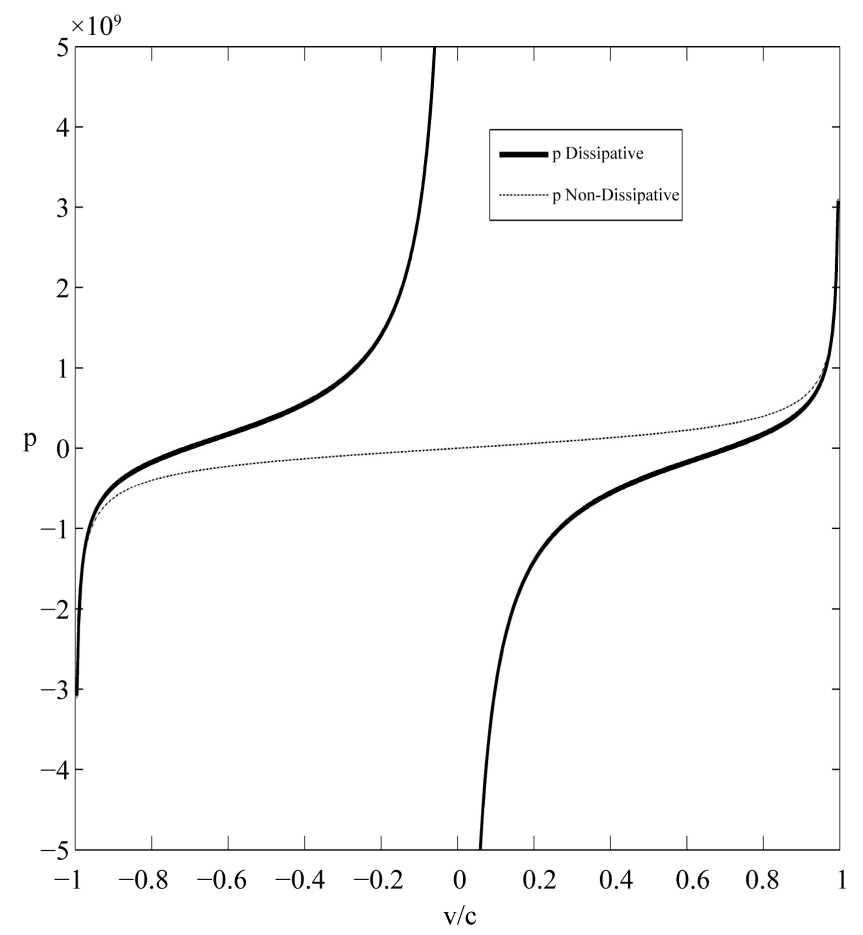

Figure 1. Relation between the generalized linear momentum and velocity.

The inverse relation of Equation (8) is shown on Figure 2, which is given analytically by

$$
\left(\frac{v}{c}\right)_{-}^{2}=\frac{1}{2}-\frac{1}{2} \frac{|p|}{\sqrt{p^{2}+4 m^{2} c^{2}}} \quad \text { if } \quad 0<\frac{v}{c} \leq \frac{1}{\sqrt{2}},
$$

and

$$
\left(\frac{v}{c}\right)_{+}^{2}=\frac{1}{2}+\frac{1}{2} \frac{|p|}{\sqrt{p^{2}+4 m^{2} c^{2}}} \quad \text { if } \quad \frac{1}{\sqrt{2}} \leq \frac{v}{c}<1 .
$$

These expressions define respectively the Hamiltonians $H^{(-)}$and $H^{(+)}$as

and

$$
H^{(-)}=\alpha c^{2} x+\frac{\sqrt{2} m_{0} c^{2}}{\left[1+\frac{|p|}{\sqrt{p^{2}+4 m^{2} c^{2}}}\right]^{1 / 2}}-m_{0} c^{2} \ln \left\{\frac{1+\frac{1}{\sqrt{2}}\left[1+\frac{|p|}{\sqrt{p^{2}+4 m^{2} c^{2}}}\right]^{1 / 2}}{\frac{1}{\sqrt{2}}\left[1-\frac{|p|}{\sqrt{p^{2}+4 m^{2} c^{2}}}\right]^{1 / 2}}\right\},
$$

$$
H^{(+)}=\alpha c^{2} x+\frac{\sqrt{2} m_{0} c^{2}}{\left[1-\frac{|p|}{\sqrt{p^{2}+4 m^{2} c^{2}}}\right]^{1 / 2}}-m_{0} c^{2} \ln \left\{\frac{1+\frac{1}{\sqrt{2}}\left[1-\frac{|p|}{\sqrt{p^{2}+4 m^{2} c^{2}}}\right]^{1 / 2}}{\frac{1}{\sqrt{2}}\left[1+\frac{|p|}{\sqrt{p^{2}+4 m^{2} c^{2}}}\right]^{1 / 2}}\right\} .
$$

\section{Trajectories}

Using the initial conditions $x=0$, with $m=1$ and $v / c=0.7$, the constant of motion (5) is determined and the trajectories on the space $(x, v)$ can be calculated. Figure 3 shows these trajectories for several values of the 


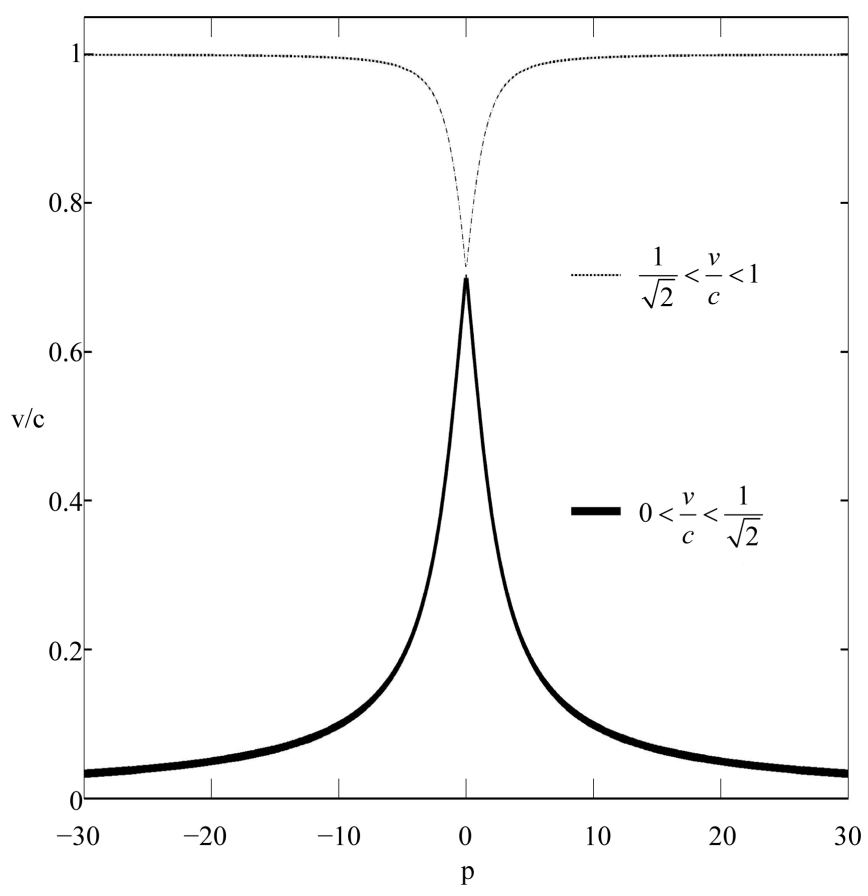

Figure 2. Inverse relation between the generalized linear momentum and velocity.

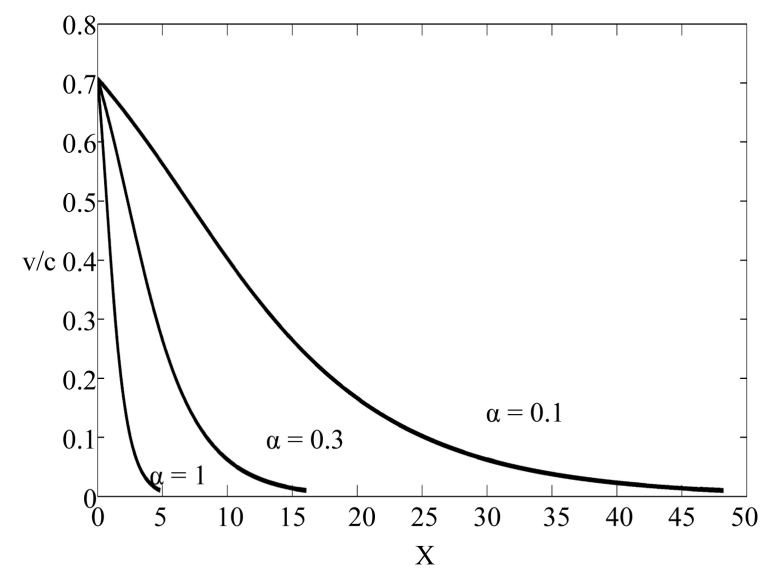

Figure 3. Trajectories in the $(x, v)$ space, defined by the constant of motion.

parameter $\alpha$. As one can see, the falling down of these trajectories and the way they are falling as the parameter $\alpha$ increases represent the behavior that one can expected for a dissipative medium. Now, given these same initial conditions, the initial generalized linear momentum is calculated from expression (8). One uses the expression (10a) to determinate the value of this Hamiltonian and to calculated the trajectories in the space $(x, p)$. These trajectories can be seen in Figure 4. As one can see, these trajectories have an odd behavior since $|p|$ go to infinity as the particle is slowing down, but this was already expected from the same expression for the generalized linear momentum, Equation (8).

\section{Conclusion}

We have constructed consistently a constant of motion, Lagrangian, and Hamiltonian for a relativistic particle moving in a dissipative medium, characterized by a force which depends on the square velocity of the particle. 


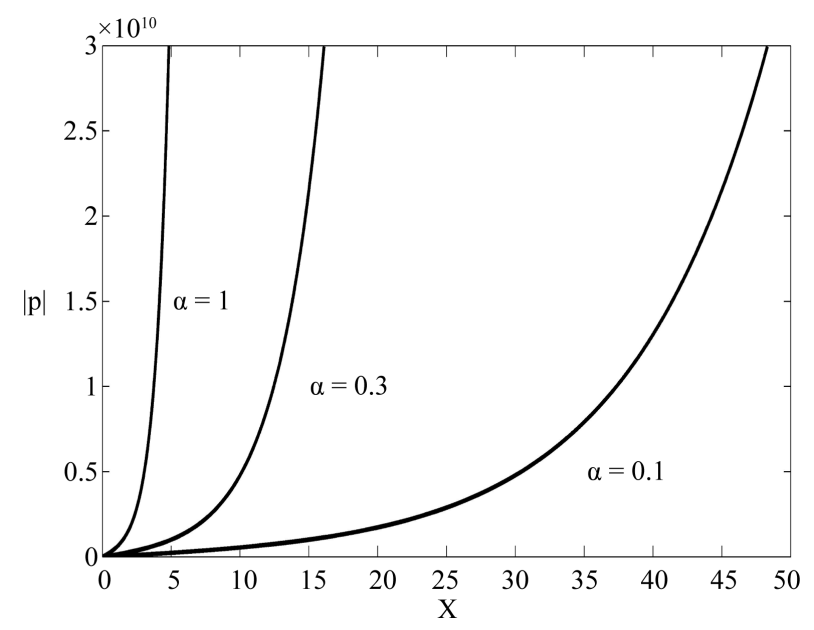

Figure 4. Trajectories in the $(x, p)$ space, defined by the Hamiltonian.

The trajectories in the space $(x, v)$, defined by the constant of motion, behave as we can expect. However, the trajectories in the space $(x, p)$, defined by the Hamiltonian, behave oddly and totally anti-intuitively. This suggests that the Hamiltonian approach applied to dissipation problem may bring about incorrect solutions if it is directly applied to quantum mechanics or statistical physics.

\section{References}

[1] Dodonov, V.P., Man’ko, V.I. and Skarzhinsky, V.D. (1981) Hadronic Journal, 4, 1734.

[2] Havas, P. (1973) Act. Phys. Austr., 38, 145.

[3] Okubo, S. (1980) Physical Review D, 22, 919. http://dx.doi.org/10.1103/PhysRevD.22.919

[4] Glauber, R. and Man'ko, V.I. (1984) Soviet Physics—JETP, 60, 450.

[5] López, G. (1996) Annals of Physics, 251, 372-383. http://dx.doi.org/10.1006/aphy.1996.0118

[6] López, G. (1998) International Journal of Theoretical Physics, 37, 1617-1623. http://dx.doi.org/10.1023/A:1026628221912

[7] López, G., López, X.E. and González, G. (2007) International Journal of Theoretical Physics, 46, 149-156. http://dx.doi.org/10.1007/s10773-006-9224-y

[8] López, G.V. (2009) arXiv:0901.4792

[9] Musielak, Z.E. (2008) Journal of Physics A: Mathematical and Theoretical, 41, Article ID: 055205. http://dx.doi.org/10.1088/1751-8113/41/5/055205

[10] Carinena, J.F. and Ranada, M.F. (2005) Journal of Mathematical Physics, 46, Article ID: 062703. http://dx.doi.org/10.1063/1.1920287

[11] López, G. (2012) Partial Differential Equations of First Order and Their Applications to Physic, 2nd Edition, World Scientific, Singapore.

[12] John, F. (1974) Partial Differential Equations. Springer-Verlag, Berlin.

[13] Kobussen, J.A. (1979) Act. Phys. Austr., 51193.

[14] Leubner, C. (1987) Physical Review A, 86, 9.

[15] Yan C.C. (1981) American Journal of Physics, 49, 296. http://dx.doi.org/10.1119/1.12632

[16] See Reference 5. 
Scientific Research Publishing (SCIRP) is one of the largest Open Access journal publishers. It is currently publishing more than 200 open access, online, peer-reviewed journals covering a wide range of academic disciplines. SCIRP serves the worldwide academic communities and contributes to the progress and application of science with its publication.

Other selected journals from SCIRP are listed as below. Submit your manuscript to us via either submit@scirp.org or Online Submission Portal.
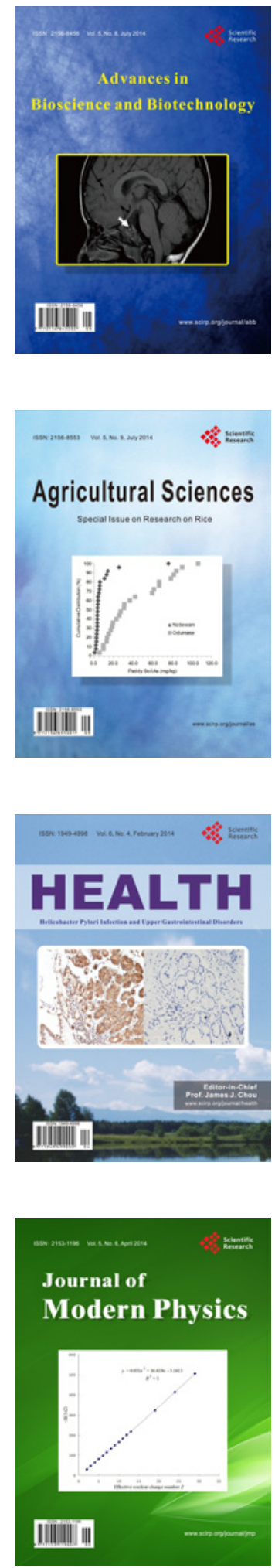
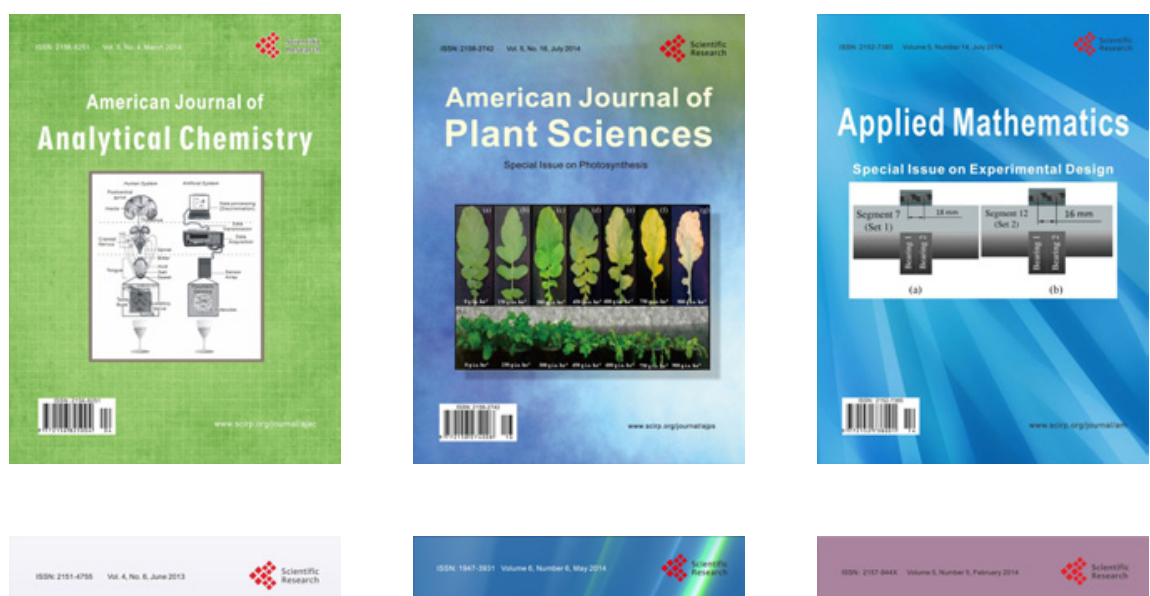

Creative Education
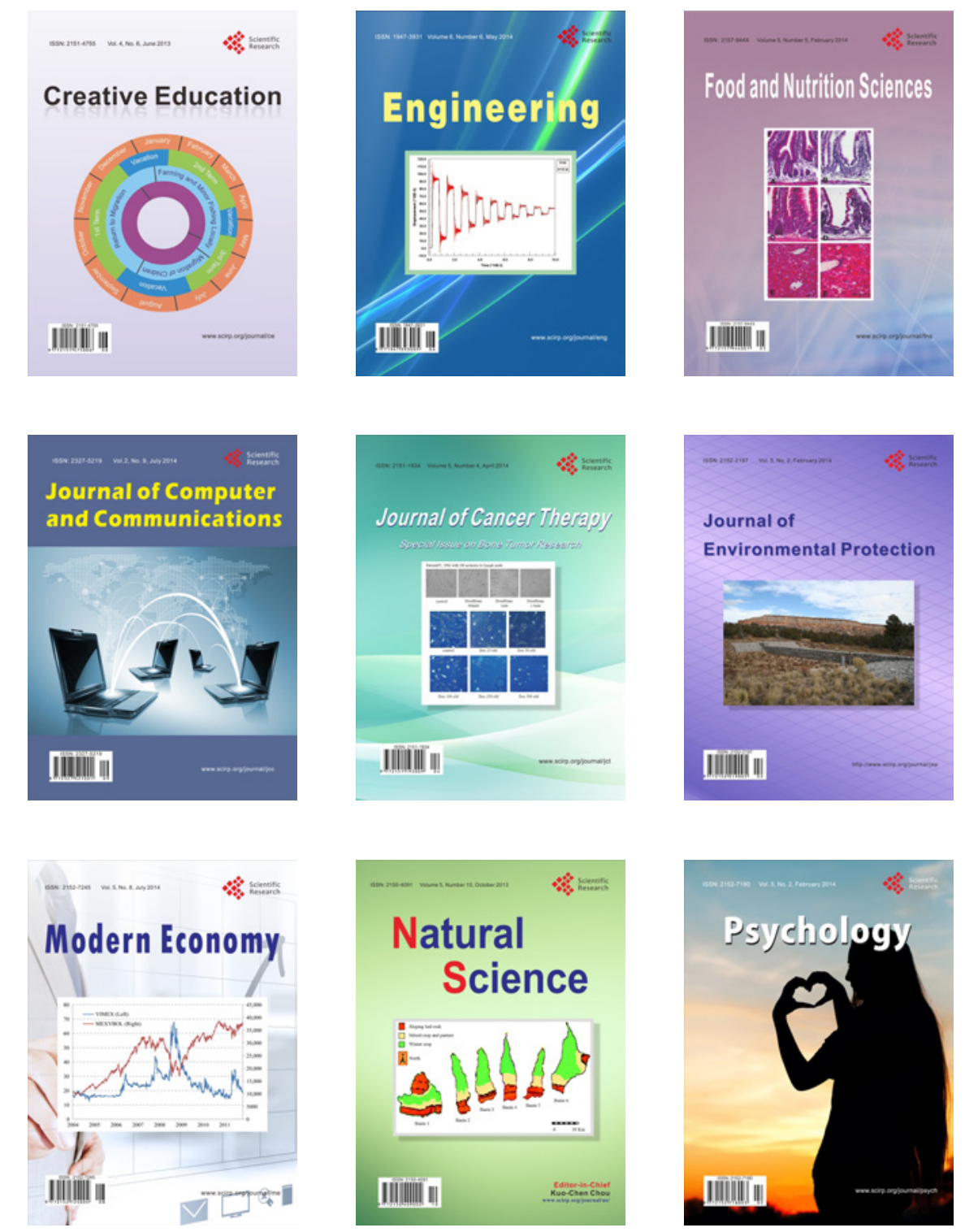